\title{
Prediction-Based Control for Nonlinear Systems with Input Delay
}

\author{
I. Estrada-Sánchez, M. Velasco-Villa, and H. Rodríguez-Cortés \\ CINVESTAV-IPN, Departamento de Ingeniería Eléctrica, Sección de Mecatrónica, Av. IPN No. 2508, Col. San Pedro Zacatenco, \\ 07360 Ciudad de México, Mexico \\ Correspondence should be addressed to M. Velasco-Villa; velasco@cinvestav.mx
}

Received 30 May 2017; Revised 4 August 2017; Accepted 27 August 2017; Published 10 October 2017

Academic Editor: Leonid Shaikhet

Copyright (C) 2017 I. Estrada-Sánchez et al. This is an open access article distributed under the Creative Commons Attribution License, which permits unrestricted use, distribution, and reproduction in any medium, provided the original work is properly cited.

\begin{abstract}
This work has two primary objectives. First, it presents a state prediction strategy for a class of nonlinear Lipschitz systems subject to constant time delay in the input signal. As a result of a suitable change of variable, the state predictor asymptotically provides the value of the state $\tau$ units of time ahead. Second, it proposes a solution to the stabilization and trajectory tracking problems for the considered class of systems using predicted states. The predictor-controller convergence is proved by considering a complete Lyapunov functional. The proposed predictor-based controller strategy is evaluated using numerical simulations.
\end{abstract}

\section{Introduction}

The importance of time delay systems has increased in the control community. Time delay systems are found in many practical problems such as chemical processes, mechanical systems, biological systems, and communication systems, for example, in teleoperation control problems. Time delays are classified as dead time, communication, or measurement delays [1]. The existence of delays in the control signal or states of the system is a major factor for closed loop stability. See, for instance, [2-4].

The control problem in time delay systems has been analyzed by several approaches, starting by ignoring its effects when the magnitude of the delay is small enough or by considering approximate predicted feedback obtained using Pade approximations for linear systems. A standard control design approach for time delayed systems is to develop prediction technics to compute the future value of the state. The estimation of future states in a dynamical system has deserved an increasing attention since the publication of the well-known Smith Predictor Compensator (SPC) [5]. The SPC solves the control problem for open-loop stable linear systems subject to time delay at the input signal. Reference [6] reports several improvements to the SPC approach. Thus, by developing state predictors, it is possible to synthesize causal state feedback for linear systems $[5,6]$.
It is mandatory first to analyze the state prediction problem, to solve, using causal state feedback, control problems for nonlinear systems with delay at the input $[7,8]$. In most of the cases, the state prediction problem is related to the pioneering work of state observation [9] or its generalization presented in [10] for delay-free nonlinear systems. The necessity of causal state feedback to solve control problems for nonlinear systems with input delay has led to developing approximate state predictors, for example, [11]. In [12] a robust controller is designed to compensate time varying perturbations through a disturbance estimator. The work [13] considers the stabilization problem for a class of linear systems with multidelays at the input. The proposed solution uses an infinite dimensional feedback based on predicted states. A sliding mode controller is presented in [14] for stabilization of systems with delayed input. A state predictor and a sliding surface are designed to minimize the effect of the delay. A robust strategy to stabilize linear input delayed systems under parametric uncertainties is presented in [15]; the solution is based on linear matrix inequalities; the same problem is addressed in [16] through a Lyapunov-Krasovskii functional. The case of linearizable feedback systems with bounded input delay is analyzed in [17] where the stabilization problem for a sufficiently small time delay is solved; the same problem for linear systems was considered in [18]. 
Another option, to tackle the control problem in time delay systems, is employing an infinite integral prediction strategy known as finite spectrum assignment proposed in [19] and its generalizations presented in [20]. Infinite integral prediction strategies are analytical solutions that suffer from practical implementation problems; see, for instance, [21] for a comparison between the infinite integral solution and a simple static state feedback. The works in [22-24] elaborate on the limitations of the finite spectrum assignment. Authors of [25] and [26] propose truncated output feedback controllers for linear systems and a class of Lipschitz nonlinear systems, respectively, to avoid implementation problems. Truncated output feedback controllers safely ignore the infinite dimensional term.

This work addresses the prediction and control problem, avoiding integral infinite dimensional solutions, for a class of nonlinear input delayed systems. Considering the linear predictor proposed in [27], this article suggests a nonlinear predictor, for a class of nonlinear systems, to compute future values of the state, $\tau$ units of time in advance. Under a Lipschitz condition assumption, it is possible to show that the proposed predictor asymptotically converges to future state values. The Lipschitz assumption is common in the literature for the delay-free nonlinear systems $[9,10]$ and for a class of nonlinear time delayed systems $[28,29]$. Additionally, the predicted future states are used to solve the trajectory tracking problem under similar assumptions to the ones for the prediction problem. The proposed predictor is equivalent to a delayed observer for an advanced system obtained using a particular change of coordinates. The convergence of prediction and trajectory tracking errors is shown by considering a complete Lyapunov-Krasovskii functional [30]. The solution proposed in this paper is close to the work in [17]. Following a different approach, the solution in [17] depends on the size of the time variant delay upper bound, while the solution developed in this paper provides an upper bound for the time delay size.

The rest of the work is organized as follows. Section 2 presents the class of dynamic systems discussed in this article. Section 3 states the prediction problem and defines the predictor dynamics. In Section 4, the solution to the trajectory tracking problem with predicted states is solved. The extension of the proposed results to full linearizable nonlinear systems is described in Section 5. Section 6 shows numerical simulations for the predictor-controller. Finally, Section 7 closes this work with concluding remarks.

\section{Class of Nonlinear Input Delay Systems}

This paper considers a class of nonlinear systems. The following differential equation describes this class of nonlinear systems:

$$
\begin{aligned}
& \dot{x}(t)=A x(t)+\Phi(x(t), u(t-\tau)), \\
& y(t)=C x(t)
\end{aligned}
$$

where $x \in X \subset \mathbb{R}^{n}$ is the state, $u(t-\tau) \in \mathbb{R}^{m}$ is the input signal, $y \in Y \subset \mathbb{R}^{m}$ is the output signal, and $\tau \in \mathbb{R}^{+}$is a constant. It is assumed that the output signal $y(t)$ is available for measurement. Moreover, nonlinear system (1) satisfies the following assumptions.

Assumption 1. $\Phi(x, u)$ is a locally Lipschitz function with respect to $x$; that is,

$$
\|\Phi(x, u)-\Phi(\widehat{x}, u)\| \leq \gamma_{L}\|x-\widehat{x}\|
$$

for all $x, \widehat{x}$ in a certain region containing the origin with $\gamma_{L} \in$ $\mathbb{R}^{+}$. In the case that (2) is satisfied for all $x, \hat{x}$, function $\Phi(x, u)$ is said to be globally Lipschitz.

Assumption 2. The pair $(A, C)$ is observable.

Notice that any nonlinear system of the form $\dot{x}=$ $f(x, u(t-\tau))$ can be expressed in the form (1) as long as $f(x, u(t-\tau))$ is differentiable with respect to $x$. A detailed justification on the generality of this class of nonlinear system (1) is found, for instance, in $[9,31,32]$.

Input delayed signals arise from the existence of deadtimes found in several industrial processes $[2,4]$ or from teleoperated systems where the control platform is placed in a remote location as could be the case of mobile robots applications [33-35].

\section{Prediction-Observation Problem}

The prediction problem is stated as follows. Based on the knowledge of the output signal $y(t)$ and the input signal $u(t)$, design a dynamic system whose states $\widehat{x}(t+\tau)$ converge asymptotically to future values of the states $x(t+\tau)$; that is,

$$
\lim _{t \rightarrow \infty}(x(t+\tau)-\widehat{x}(t+\tau))=0 .
$$

To motivate the importance of the prediction problem, assume that there exists a state feedback controller

$$
u(t)=\alpha(x(t), t)
$$

that solves the trajectory tracking problem for system (1) with $\tau=0$. Due to the involved time delay, the closed loop dynamics (1)-(4) took the following form:

$$
\dot{x}(t)=A x(t)+\Phi(x(t), \alpha(x(t-\tau), t-\tau)) ;
$$

thus, in general, the state feedback controller (4) no longer solves the trajectory tracking problem for (1). Assume that it is possible to design an ideal feedback based on future values of the state, this feedback, in terms of future states, will take the form

$$
u(t)=\alpha(x(t+\tau), t+\tau) .
$$

Notice that the use of the ideal feedback (6) will produce ideal delay-free closed loop dynamics (1)-(6). This fact motivates the design of a predictor capable of generating the estimated value $\widehat{x}(t+\tau)$. However, as expected, the best possible solution only achieves asymptotic convergence to future states. Moreover, due to the time delay effect, the closed loop dynamics will behave as in open-loop on $[0, \tau]$ producing an undesirable and not compensable transient. 
Instrument in addressing the prediction problem for the class of systems described in (1) is the following change of coordinates:

$$
\begin{aligned}
& w(t)=x(t+\tau), \\
& x(t)=w(t-\tau) .
\end{aligned}
$$

In terms of new coordinates (7), dynamic system (1) is described by the following equations:

$$
\begin{aligned}
\dot{w}(t) & =A w(t)+\Phi(w(t), u(t)), \\
y(t) & =C w(t-\tau) .
\end{aligned}
$$

This dynamic system represents the evolution of original system (1) and $\tau$ units of time ahead.

3.1. Predictor Design. The predictor dynamics can now be designed based on time advanced system (8). The dynamics for the predictor is proposed as a copy of the time advanced dynamics (8) plus a term injecting the delayed observer error:

$$
\begin{aligned}
\dot{\widehat{w}}(t)= & A \widehat{w}(t)+\Phi(\widehat{w}(t), u(t)) \\
& +L C[x(t)-\widehat{w}(t-\tau)],
\end{aligned}
$$

where $L$ is the predictor matrix gain, and $\widehat{w}(t)$ represents the predicted evolution of the state $w(t)$ and consequently the prediction of $\widehat{x}(t+\tau)$ that represent the original state $x(t) \tau$ time units ahead. Notice that, in (9), it is not possible to inject the signal $w(t)$ since according to (7) this is not available.

To analyze the predictor convergence of the state $\widehat{w}(t)$ to the future value $w(t)=x(t+\tau)$ notice that from the systempredictor dynamic (1)-(9) it is possible to define the error signal:

$$
e_{w}(t)=x(t)-\widehat{w}(t-\tau)
$$

The time derivative of (10) produces

$$
\dot{e}_{w}(t)=A e_{w}(t)-L C e_{w}(t-\tau)+\widetilde{\Phi}(x, \widehat{x}, u)
$$

with $\widetilde{\Phi}(x, \widehat{x}, u)=\Phi(x(t), u(t-\tau))-\Phi(\widehat{x}(t), u(t-\tau))$.

3.2. Predictor Convergence. The convergence to the origin of the prediction error (10) is proven in two steps. First, notice that the stability of the perturbation-free system (11) corresponds to the stability of a linear system of the form

$$
\begin{aligned}
& \dot{\psi}(t)=A \psi(t)+A_{1} \psi(t-\tau), \\
& \psi(\theta)=\varphi(\theta)
\end{aligned}
$$

with an initial condition function $\varphi(\theta), \theta \in[-\tau, 0]$ with $\varphi \in$ $\mathscr{P} \mathscr{C}\left([-\tau, 0], \mathbb{R}^{n}\right)$, the space of piecewise continuous functions of $\mathbb{R}^{n}$ defined in $[-\tau, 0]$.

Notice that the prediction error initial condition depends on those of plant (1) and predictor (9). Since the parameters of matrix $L$ are free, from the observability assumption of the pair $(A, C)$, it is always possible to locate the eigenvalues of the matrix $A-L C$ in the left half complex plane. The Hurwitz property of matrix $A-L C$ ensures the stability of differential difference equation (12) for a sufficiently small time delay $\tau$ [21]. The maximum time delay $\tau^{*}$ for which system (12) will be asymptotically stable depends on the choice of the observer gain $L$ and its computation is not an easy task since its complexity rises as the dimension of system (12) increases. Thus, given a matrix gain $L$, such that $A-L C$ is a Hurwitz matrix, it is always possible to asymptotically stabilize prediction error (12) for a constant time delay $\tau<$ $\tau^{*}$. This fact can be verified, for instance, by considering Proposition 5.2.2, Chapter 5 in [36], or reviewing the pole assignment procedure given in [21].

Based on the above arguments, it will be assumed, without loss of generality, that there exist $L$ and $\tau^{*}$ such that system (12) is asymptotically stable for $0 \leq \tau<\tau^{*}$. It should also be noticed that the stability of (12) is not only asymptotic but also exponential [37].

3.2.1. Complete Type Functional. Following the results presented in [30], to show the asymptotic stability of (11), it is possible to consider a complete type functional of the form

$$
\begin{aligned}
V\left(e_{w t}\right)= & V_{0}\left(e_{w t}\right) \\
& +\int_{-\tau}^{0} e_{w}^{\top}(t+\theta)\left[(\tau+\theta) W_{1}\right] e_{w}(t+\theta) d \theta,
\end{aligned}
$$

where $e_{w t}=e_{w}(t-\tau), \theta \in[-\tau, 0]$ with

$$
\begin{aligned}
& V_{0}\left(e_{w t}\right)=e_{w}^{\top}(t) U(0) e_{w}(t)+2 e_{w}^{\top}(t) \int_{-\tau}^{0} U(-\tau-\theta) \\
& \cdot A_{1} e_{w}(t+\theta) d \theta+\int_{-\tau}^{0} e_{w}^{\top}\left(t+\theta_{1}\right) \\
& \cdot A_{1}^{\top}\left[\int_{-\tau}^{0} U\left(\theta_{1}-\theta_{2}\right) A_{1} e_{w}\left(t+\theta_{2}\right) d \theta_{2}\right] d \theta_{1}
\end{aligned}
$$

and $A_{1}=-L C$. The Lyapunov matrix $U(\cdot)$ in (14) is given by

$$
U(\gamma)=\int_{0}^{\infty} K^{\top}(t) W K(t+\gamma) d t
$$

where $K(t)$ is the fundamental matrix of system (12) without perturbation, and

$$
W=W_{0}+\tau W_{1}
$$

with positive definite matrices $W_{0}$ and $W_{1}$.

The Lyapunov matrix $U(\gamma)$ satisfies the following properties [30].

(P1) $d U(\gamma) / d \gamma=U(\gamma) A+U(\gamma-\tau) A_{1}$.

(P2) $U(-\gamma)=U^{\top}(\gamma), \gamma>0$ and $U^{\top}(0)=U(0)$.

(P3) $U(0) A+\mathrm{U}(-\tau) A_{1}+A^{\top} U(0)+A_{1}^{\top} U(\tau)=-W$.

It is possible now to state the prediction convergence result. 
Theorem 3. Suppose that the matrix gain L is chosen such that perturbation-free system (12) is exponentially stable. Consider that Assumption 1 is satisfied, the prediction error $e_{w}(t)$ converges asymptotically to the origin if

$$
\begin{aligned}
& \text { (i) } \tau<\frac{\lambda_{\min }\left(W_{0}\right)}{\lambda_{\min }\left(W_{1}\right)}-\frac{2}{a_{1}}, \\
& \text { (ii) } \lambda_{\min }\left(W_{1}\right)>\gamma_{L} v a_{1},
\end{aligned}
$$

where $v=\max _{\theta \in[0, \tau]}\|U(\theta)\|$, and $a_{1}=\sqrt{\lambda_{\max }\left(A_{1}^{\top} A_{1}\right)}$ with $\lambda_{\min }(\cdot)\left(\lambda_{\max }(\cdot)\right)$, the smaller (largest) eigenvalue of $(\cdot)$. As a consequence,

$$
\lim _{t \rightarrow \infty}(\widehat{w}(t)-x(t+\tau))=0 .
$$

Moreover, if $\Phi(x, u)$ in Assumption 1 is globally Lipschitz, the resulting convergence is also global.

Proof. Considering the properties of the Lyapunov matrix $U(\gamma)$, after some manipulations, the time derivative of functional (13), along the solution of the prediction error (11), produces

$$
\begin{aligned}
\frac{d}{d t} V & \left(e_{w t}\right) \\
= & -e_{w}^{\top}(t) W_{0} e_{w}(t)-\int_{t-\tau}^{t} e_{w}^{\top}(\xi) W_{1} e_{w}(\xi) d \xi \\
& +2 e_{w}^{\top}(t) U(0) \widetilde{\Phi}(\cdot) \\
& +2 \widetilde{\Phi}^{\top}(\cdot) \int_{t-\tau}^{t} U(-\tau-\xi+t) A_{1} e_{w}(\xi) d \xi
\end{aligned}
$$

Then, it is possible to upper-bound the undefined sign terms in (19) as follows:

$$
\begin{aligned}
& 2 e_{w}^{\top}(t) U(0) \widetilde{\Phi}(\cdot) \leq 2 \gamma_{L} v\left\|e_{w}(t)\right\|^{2}, \\
& 2 \widetilde{\Phi}^{\top}(\cdot) \int_{t-\tau}^{t} U(-\tau-\xi+t) A_{1} e_{w}(\xi) d \xi \\
& \quad \leq \gamma_{L} v a_{1} \tau\left\|e_{w}(t)\right\|^{2}+\gamma_{L} v a_{1} \int_{-\tau}^{0}\left\|e_{w}(t+\theta)\right\|^{2} d \theta .
\end{aligned}
$$

Therefore, (19) can be upper-bounded in the following form:

$$
\begin{aligned}
\frac{d}{d t} V\left(e_{w t}\right) \leq & -e_{w}^{\top}(t) W_{0} e_{w}(t) \\
& -\int_{-\tau}^{0} e_{w}^{\top}(t+\theta) W_{1} e_{w}(t+\theta) d \theta \\
& +2 \gamma_{L} v\left\|e_{w}(t)\right\|^{2}+\gamma_{L} v a_{1} \tau\left\|e_{w}(t)\right\|^{2} \\
& +\gamma_{L} v a_{1} \int_{-\tau}^{0}\left\|e_{w}(t+\theta)\right\|^{2} d \theta .
\end{aligned}
$$

As a result, one has

$$
\begin{array}{rl}
\frac{d}{d t} V & V\left(e_{w t}\right) \\
\leq & -\left[\lambda_{\min }\left(W_{0}\right)-\left(2+a_{1} \tau\right) \gamma_{L} v\right]\left\|e_{w}(t)\right\|^{2} \\
& -\left[\lambda_{\min }\left(W_{1}\right)-\gamma_{L} v a_{1}\right] \int_{-\tau}^{0}\left\|e_{w}(t+\theta)\right\|^{2} d \theta .
\end{array}
$$

Notice that condition (i) can be rewritten as

$$
\lambda_{\min }\left(W_{0}\right)>\left(2+a_{1} \tau\right) \gamma_{L} v\left(\frac{\lambda_{\min }\left(W_{1}\right)}{\gamma_{L} v a_{1}}\right) .
$$

Then, one obtains

$$
\lambda_{\min }\left(W_{0}\right)>\left(2+a_{1} \tau\right) \gamma_{L} v
$$

since

$$
\frac{\lambda_{\min }\left(W_{1}\right)}{\gamma_{L} v a_{1}}>1
$$

from condition (ii). Conditions (24) and (25) conclude the proof. Finally, notice that since the perturbation-free system (11) is globally stabilizable, if $\Phi(x, u)$ is globally Lipschitz with respect to $x$, the result will be also global.

Remark 4. For delay-free systems of form (1), that is,

$$
\begin{aligned}
& \dot{\xi}=A \xi+\Phi(\xi, u(t)), \\
& y=C \xi,
\end{aligned}
$$

predictor (9) (with $\tau=0$ ) becomes the classical Luenberger observer for system (26). In this case, from (7), $w(t)=\xi(t)$. Following [9], if the matrix gain $L$ is chosen such that

$$
\gamma_{L}<\frac{\lambda_{\min }(Q)}{2 \lambda_{\max }(P)}
$$

where $P$ and $Q$ are symmetric, positive definite matrices associated with

$$
(A-L C)^{\top} P+P(A-L C)=-Q,
$$

then, observer (9) (with $\tau=0$ ) converges asymptotically to the real states, $w(t)=\xi(t)$. The optimal value of $\gamma_{L}$ is obtained for $Q=I$, [38]. Thus, the observation problem is reduced to choose $L$ such that

$$
\gamma_{L}<\frac{1}{2 \lambda_{\max }(P)} .
$$

Further analysis of this problem has been carried out in [31, 32].

Remark 5. Notice also that, in the case of $\tau=0$, property (P3) takes the form

$$
\left(A+A_{1}\right)^{\top} U(0)+U(0)\left(A+A_{1}\right)=-W .
$$




\section{Control Problem Based on Predicted State Feedback}

The regulation or trajectory tracking problem for time delayed systems, based on predicted states, is more challenging than the prediction problem. First, the prediction errors link up with the regulation or trajectory tracking errors. Second, if the matching condition between the disturbances and the control input is not satisfied control design has to be addressed case by case. To illustrate the use of the proposed state predictor for control design a narrow class of nonlinear systems is considered. It is assumed that $\Phi(x, u(t-\tau))$ has the structure

$$
\Phi(x, u(t-\tau))=B\left(u(t-\tau)+\Phi_{1}(x)\right)
$$

which satisfies Assumption 1. It is also considered that the following assumption holds.

Assumption 6. The pair $(A, B)$ associated with system (1) is controllable.

Based on the state predictor (9) it is possible to propose the following state feedback:

$$
u(t)=k \widehat{w}(t)-\Phi_{1}(\widehat{w}) .
$$

From (10), the closed loop system (1)-(4), (6)-(16), (19), (22), (24)-(29), and (31)-(32) produces

$$
\begin{aligned}
\dot{x}(t) & =(A+B k) x(t)-B k e_{w}(t)+B \widetilde{\Phi}_{1}(x, \widehat{x}), \\
\dot{e}_{w}(t) & =A e_{w}(t)-L C e_{w}(t-\tau)+B \widetilde{\Phi}_{1}(x, \widehat{x})
\end{aligned}
$$

for $\widetilde{\Phi}_{1}(x, \widehat{x})=B\left(\Phi_{1}(x)-\Phi_{1}(\widehat{x}(t))\right.$. From Assumptions 2 and 6 , the matrices $A+B k$ and $A-L C$ are chosen, through the selection of $L$ and $k$, to be Hurwitz. System (33) can be rewritten as

$$
\dot{\zeta}(t)=A_{r} \zeta(t)+A_{1 r} \zeta(t-\tau)+\widetilde{\Phi}_{r}(x, \widehat{x})
$$

for $\zeta=\left[x, e_{w}\right]^{\top}$ and

$$
\begin{aligned}
A_{r} & =\left[\begin{array}{cc}
A+B k & -B k \\
0 & A
\end{array}\right], \\
A_{1 r} & =\left[\begin{array}{cc}
0 & 0 \\
0 & -L C
\end{array}\right], \\
\widetilde{\Phi}_{r} & =\left[\begin{array}{l}
B \widetilde{\Phi}_{1}(x, \widehat{x}) \\
B \widetilde{\Phi}_{1}(x, \widehat{x})
\end{array}\right] .
\end{aligned}
$$

Notice that system (34) has exactly the structure of system (11); this fact allows adapting the statement of Theorem 3 to give a solution to the state predictor-based stabilization problem.

From the choice of gains $L$ and $k$, it is clear that matrix $A_{r}+A_{1 r}$ is also Hurwitz. From Assumption 1, function $\widetilde{\Phi}_{r}(x, \widehat{x})$ is a Lipschitz function with respect to $\zeta(t)$; that is,

$$
\left\|\widetilde{\Phi}_{r}(x, \widehat{x})\right\| \leq \gamma_{r}\|\zeta\| .
$$

Therefore, it is possible to state the solution to the state prediction-based stabilization problem in the next theorem.

Theorem 7. Suppose that L in (9) and $k$ in (32) are chosen such that the perturbation-free system (34) is exponentially stable. Then, the prediction-based stabilization problem for system (1)-(31) has a solution if

$$
\begin{aligned}
& \text { (i) } \tau<\frac{\lambda_{\min }\left(W_{0 r}\right)}{\lambda_{\min }\left(W_{1 r}\right)}-\frac{2}{a_{1 r}}, \\
& \text { (ii) } \lambda_{\min }\left(W_{1 r}\right)>\gamma_{r} v_{r} a_{1 r}
\end{aligned}
$$

for $v_{r}=\max _{\theta \in[0, \tau]}\left\|U_{r}(\theta)\right\|, a_{1 r}=\sqrt{\lambda_{\max }\left(A_{1 r}^{\top} A_{1 r}\right)}$, and positive definite matrices $W_{0 r}$ and $W_{1 r}$.

Proof. The proof of this theorem follows the same lines of Theorem 3. The complete Lyapunov functional is constructed with $A_{r}, A_{1 r}$, and

$$
\begin{gathered}
W_{r}=W_{0 r}+\tau W_{1 r}, \\
U_{r}(\gamma)=\int_{0}^{\infty} K_{r}^{\top}(t) W_{r} K_{r}(t+\gamma) d t
\end{gathered}
$$

to build the fundamental matrix $K_{r}(t)$ associated with the perturbation-free system, that is, system (34) with $\widetilde{\Phi}_{r}(x, \widehat{x})=$ 0 . The new Lyapunov matrix $U_{r}(\gamma)$ also satisfies the properties (P1), (P2), and (P3) taking care of the corresponding subscripts associated with system (34).

\section{Full Linearizable Systems Case}

To strengthen the importance of the class of nonlinear systems (1), notice a broad class of nonlinear systems of the form

$$
\begin{gathered}
\dot{x}=f(x)+\sum_{j=1}^{m} g_{i}(x) u_{i}(t-\tau), \\
y_{1}=h_{1}(x), \ldots, y_{m}=h_{m}(x),
\end{gathered}
$$

where $x \in \mathscr{X} \subset \mathbb{R}^{n}, u \in \mathbb{R}^{m}, y \in \mathbb{R}^{m}$, smooth vector fields $f, g_{i}: \mathbb{R}^{n} \rightarrow \mathbb{R}^{n}, i=1, \ldots, m$, and scalar valued functions $h_{j}, j=1, \ldots, m$ from $\mathbb{R}^{n}$ to $\mathbb{R}$ can be transformed to a system of form (1) as long as system (39) has a well-defined relative degree $n=r_{1}+\cdots+r_{m}$ (see, [39]).

The transformation of system (39) to a set of systems of form (1) can be done through the diffeomorphism, $\xi(t)=$ $\phi(x(t)), \xi(0)=\phi(x(0))$ with

$$
\begin{aligned}
\phi(x) & =\left[\phi_{1}(x) \cdots \phi_{m}(x)\right]^{\top}, \\
\phi_{i}(x) & =\left[h_{i}(x), L_{f} h_{i}(x), \ldots, L_{f}^{r_{i}-1} h_{i}(x)\right]^{\top}, \\
& i=1, \ldots, m .
\end{aligned}
$$


As a result, system (39) can be rewritten in the form

$$
\begin{gathered}
\dot{\xi}_{i 1}=\xi_{i 2} \\
\vdots \\
\dot{\xi}_{i r_{i}-1}=\xi_{i r_{i}} \\
\dot{\xi}_{i r_{i}}=b_{i}(\xi)+\sum_{j=1}^{m} a_{i j}(\xi) u_{j}(t-\tau) \\
y_{i}=\xi_{i 1}
\end{gathered}
$$

with

$$
\begin{aligned}
b_{i}(\xi) & =\left.L_{f}^{r_{i}} h_{i}(x)\right|_{x=\phi^{-1}(\xi)} \\
a_{i j}(\xi) & =\left.L_{g_{j}} L_{f}^{r_{i}-1} h_{i}(x)\right|_{x=\phi^{-1}(\xi)}
\end{aligned}
$$

for $i=1, \ldots, m$ where $a_{i j}(\xi)$ are entries of the invertible decoupling matrix $A_{d}(x)$ [39]. It is clear that system (41) can be assumed to have the structure given by (1).

Notice that, in the case of system (39), defining

$$
\omega(t)=\xi(t+\tau)
$$

the time ahead representation of (41) is obtained as

$$
\begin{gathered}
\dot{\omega}_{i 1}=\omega_{i 2} \\
\vdots \\
\dot{\omega}_{i r_{i}-1}=\omega_{i r_{i}} \\
\dot{\omega}_{i r_{i}}=b_{i}(\omega)+\sum_{j=1}^{m} a_{i j}(\omega) u_{j}(t) \\
y_{\omega i}=\omega_{i 1}
\end{gathered}
$$

for $i=1, \ldots, m$.

The predictor for system (44) takes the form

$$
\begin{gathered}
\dot{\widehat{\omega}}_{i 1}=\widehat{\omega}_{i 2}+\lambda_{i r_{i}-1}\left(\xi_{i 1}(t)-\widehat{\omega}_{i 1}(t-\tau)\right) \\
\vdots \\
\dot{\hat{\omega}}_{i r_{i}-1}=\widehat{\omega}_{i r_{i}}+\lambda_{i 1}\left(\xi_{i 1}(t)-\widehat{\omega}_{i 1}(t-\tau)\right) \\
\dot{\widehat{\omega}}_{i r_{i}}=b_{i}(\widehat{\omega})+\sum_{j=1}^{m} a_{i j}(\widehat{\omega}) u_{j}(t) \\
+\lambda_{i 0}\left(\xi_{i 1}(t)-\widehat{\omega}_{i 1}(t-\tau)\right) \\
y_{\omega i}=\omega_{i 1} .
\end{gathered}
$$

Defining the prediction errors as

$$
e_{\omega_{i j}}(t)=\xi_{i j}(t)-\widehat{\omega}_{i j}(t-\tau)=\xi_{i j}(t)-\widehat{\xi}_{i j}(t)
$$

for $i=1, \ldots, m$ and $j=1, \ldots, r_{i}$ allows writing the prediction error dynamics in the form

$$
\begin{gathered}
\dot{e}_{\omega_{i 1}}=e_{\omega_{i 2}}-\lambda_{i r_{i}-1} e_{\omega_{i 1}}(t-\tau) \\
\vdots \\
\dot{e}_{\omega_{i r_{i}-1}}=e_{\omega_{i r_{i}}}-\lambda_{i 1} e_{\omega_{i 1}}(t-\tau) \\
\dot{e}_{\omega_{i r_{i}}}=\left[b_{i}(\xi)-b_{i}(\widehat{\xi})\right] \\
\quad+\sum_{j=1}^{m}\left[a_{i j}(\xi)-a_{i j}(\widehat{\xi})\right] u_{j}(t-\tau)-\lambda_{i 0} e_{\omega_{i 1}}(t-\tau) .
\end{gathered}
$$

Remark 8. Notice that for the input-state linearizable system (39), due to the change of coordinates $w(t)=\xi(t+\tau)$ and the diffeomorphism, $\xi(t+\tau)=\phi(x(t+\tau))$, the estimated future value is obtained as

$$
\widehat{x}(t+\tau)=\phi^{-1}(\widehat{w}(t)) .
$$

5.1. Control Problem for Full Linearizable Systems. For control design in the case of full linearizable systems, the dynamics described by the set of differential equations in (39) is considered, or equivalently, a system of form (41) which has the structure of (1).

A feedback proposed for the solution of the delay-free case [39] can be modified based on future states as

$$
u(t)=A_{d}^{-1}(\widehat{\omega})\{-b(\widehat{\omega})+v(t)\},
$$

where the entries of matrix $A_{d}(\widehat{\omega})$ and vector $b(\widehat{\omega})=$ $\left[b_{1} \cdots b_{m}\right]^{\top}$ are given in (42) and the new input signal $v=$ $\left[v_{1} \cdots v_{m}\right]^{\top}$ is defined as

$$
\begin{aligned}
v_{i}(t)= & \dot{\omega}_{i r_{i}}^{d}-k_{r_{i}-1}\left(\widehat{\omega}_{i r_{i}-1}-\omega_{i r_{i}-1}^{d}\right)-\cdots \\
& -k_{0}\left(\widehat{\omega}_{i 1}-\omega_{i 1}^{d}\right)
\end{aligned}
$$

with $i=1, \ldots, m$ where $\omega_{i j}^{d}(t)=\xi_{i j}^{d}(t+\tau)$ is the desired reference for $\omega_{i j}(t)$.

Notice that in the case of a stabilization problem for system (39) and (41), feedback (49) is rewritten considering $\omega_{i j}^{d}=0$ for $i=1, \ldots, m$ and $j=1, \ldots, r_{i}$.

The closed loop system (41), (42), (44)-(47), and (49) produces

$$
\begin{aligned}
& {\left[\begin{array}{c}
\dot{\xi}_{1 r_{1}} \\
\vdots \\
\dot{\xi}_{m r_{m}}
\end{array}\right]=b(\xi)+A_{d}(\xi) A_{d}^{-1}(\widehat{\omega}(t-\tau))} \\
& \quad \cdot\{-b(\widehat{\omega}(t-\tau))+v(t-\tau)\}=v(t-\tau) \\
& +[b(\xi)-b(\widehat{\omega}(t-\tau))] \\
& +\left[A_{d}(\xi) A_{d}^{-1}(\widehat{\omega}(t-\tau))-I\right] \\
& \cdot\{-b(\widehat{\omega}(t-\tau))+v(t-\tau)\}
\end{aligned}
$$


where

$$
\begin{aligned}
v_{i}(t-\tau)= & \dot{\xi}_{i r_{i}}^{d}-k_{r_{i}-1}\left(\widehat{\xi}_{i r_{i}-1}-\xi_{i r_{i}-1}^{d}\right)-\cdots \\
& -k_{0}\left(\widehat{\xi}_{i 1}-\xi_{i 1}^{d}\right) .
\end{aligned}
$$

Defining a set of tracking errors as

$$
e_{d_{i j}}(t)=\xi_{i j}(t)-\xi_{i j}^{d}(t)
$$

the following is obtained:

$$
\begin{aligned}
v_{i}(t-\tau)= & \dot{\xi}_{i r_{i}}^{d}-k_{r_{i}-1}\left(e_{d i r_{i}-1}-e_{\omega i r_{i}-1}\right)-\cdots \\
& -k_{0}\left(e_{d i 1}-e_{\omega i 1}\right) \\
= & \dot{\xi}_{i r_{i}}^{d}-\sum_{j=1}^{r_{i}-1} k_{j} e_{d_{i j}}+\sum_{j=1}^{r_{i}-1} k_{j} e_{\omega_{i j}}
\end{aligned}
$$

and, as a result, one has

$$
\begin{aligned}
{\left[\begin{array}{c}
\dot{e}_{d_{1 r_{1}}}(t) \\
\vdots \\
\dot{e}_{d_{m r_{m}}}(t)
\end{array}\right]=} & {\left[\begin{array}{c}
-\sum_{j=1}^{r_{1}-1} k_{j} e_{d_{1 j}} \\
\vdots \\
r_{m}-1 \\
-\sum_{j=1} k_{j} e_{d_{m j}}
\end{array}\right]+\left[\begin{array}{c}
\sum_{j=1}^{r_{1}-1} k_{j} e_{\omega_{1 j}} \\
\vdots \\
\sum_{j=1}^{r_{m}-1} k_{j} e_{\omega_{m j}}
\end{array}\right] } \\
& +\chi(t),
\end{aligned}
$$

where

$$
\begin{aligned}
\chi(t)= & {[b(\xi)-b(\widehat{\xi})] } \\
& +\left[A_{d}(\xi) A_{d}^{-1}(\widehat{\xi})-I\right]\{-b(\widehat{\xi})+v(t-\tau)\} .
\end{aligned}
$$

Therefore, replacing the feedback (49) with (47), the complete closed loop dynamics is given by

$$
\begin{gathered}
\dot{e}_{d_{i 1}}=e_{d_{i 2}} \\
\vdots \\
\dot{e}_{d_{i_{i}-1}}=e_{d_{i_{i}}} \\
\dot{e}_{i_{i_{i}}}=-\sum_{j=1}^{r_{i}-1} k_{i j} e_{d_{i j}}+\sum_{j=1}^{r_{i}-1} k_{i j} e_{\omega_{i j}}+\chi_{i}, \\
\dot{e}_{\omega_{i 1}}=e_{\omega_{i 2}}-\lambda_{r_{i}-1} e_{\omega_{i 1}}(t-\tau) \\
\vdots \\
\dot{e}_{\omega_{i_{i}-1}}=e_{\omega_{i_{i}}}-\lambda_{1} e_{\omega_{i 1}}(t-\tau) \\
\dot{e}_{\omega_{i_{i}}}=-\lambda_{0} e_{\omega_{i 1}}(t-\tau)+\chi_{i},
\end{gathered}
$$

where $\chi_{i}$ corresponds to the $i$-row of vector $\chi$.
System (57) can be rewritten in a vector form as follows:

$$
\begin{aligned}
& \dot{e}_{d_{i}}(t)=A_{e_{d} i} e_{d_{i}}(t)+B_{e_{d} i} e_{\omega_{i}}(t)+\gamma_{i}, \\
& \dot{e}_{w_{i}}(t)=A_{e_{w i} i} e_{w_{i}}(t)+B_{e_{w i}} e_{\omega_{i}}(t-\tau)+\gamma_{i}
\end{aligned}
$$

with

$$
\begin{aligned}
A_{e_{d} i} & =\left[\begin{array}{ccccc}
0 & 1 & 0 & \cdots & 0 \\
0 & 0 & 1 & \cdots & 0 \\
\vdots & & & \ddots & \vdots \\
0 & \cdots & \cdots & 0 & 1 \\
-k_{i 1} & -k_{i 2} & \cdots & \cdots & -k_{i r_{i}-1}
\end{array}\right], \\
B_{e_{d} i} & =\left[\begin{array}{ccccc}
0 & 0 & 0 & \cdots & 0 \\
0 & 0 & 0 & \cdots & 0 \\
\vdots & & & \ddots & \vdots \\
0 & \cdots & \cdots & 0 & 0 \\
k_{i 1} & k_{i 2} & \cdots & \cdots & k_{i r_{i}-1}
\end{array}\right] \\
A_{e_{w} i} & =\left[\begin{array}{ccccc}
0 & 1 & 0 & \cdots & 0 \\
0 & 0 & 1 & \cdots & 0 \\
\vdots & & & \ddots & \vdots \\
0 & \cdots & \cdots & 0 & 1 \\
0 & 0 & \cdots & \cdots & 0
\end{array}\right]
\end{aligned}
$$

$$
B_{e_{w i} i}=\left[\begin{array}{ccccc}
-\lambda_{i r_{i}-1} & 0 & 0 & \cdots & 0 \\
\vdots & 0 & 0 & \cdots & 0 \\
\vdots & & & \ddots & \vdots \\
-\lambda_{i 1} & \cdots & \cdots & 0 & 0 \\
-\lambda_{i 0} & 0 & \cdots & \cdots & 0
\end{array}\right]
$$

and $\gamma_{i}=\left[0, \ldots, 0, \chi_{i}\right]^{\top}$. Therefore, the following can be written:

$$
e_{i}(t)=A_{e_{i}} e_{i}(t)+B_{e_{i}} e_{i}(t-\tau)+\Gamma_{i},
$$

where

$$
\begin{aligned}
e_{i} & =\left[\begin{array}{l}
e_{d_{i}} \\
e_{\omega_{i}}
\end{array}\right], \\
A_{e_{i}} & =\left[\begin{array}{cc}
A_{e_{d} i} & B_{e_{d} i} \\
0 & A_{e_{w} i}
\end{array}\right], \\
B_{e_{i}} & =\left[\begin{array}{cc}
0 & 0 \\
0 & B_{e_{w} i}
\end{array}\right], \\
\Gamma_{i} & =\left[\begin{array}{l}
\gamma_{i}(t) \\
\gamma_{i}(t)
\end{array}\right] .
\end{aligned}
$$




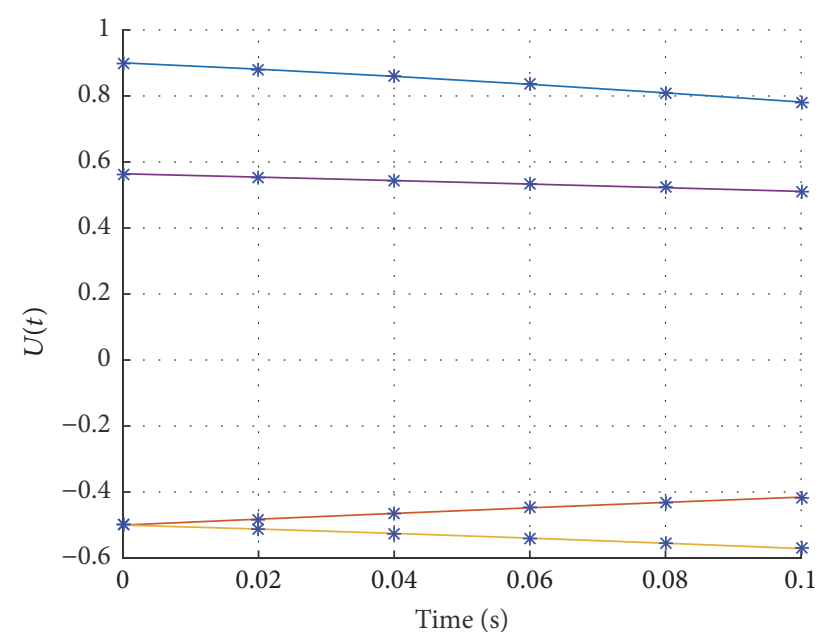

FIgURE 1: Evolution of matrix $U(t)$.

Finally, one has

$$
\dot{e}=A_{e} e(t)+B_{e} e(t-\tau)+\Gamma(t),
$$

where $e=\left[e_{1} \cdots e_{m}\right]^{\top}, A_{e}=\operatorname{diag}\left\{A_{e_{i}}\right\}, B_{e}=\operatorname{diag}\left\{B_{e_{i}}\right\}$, and $\Gamma(t)=\left[\Gamma_{1} \cdots \Gamma_{m}\right]^{\top}$.

Based on the above developments, the trajectory tracking problem for system (39) and (41) based on predicted future values is stated under the same lines of Theorems 3 and 7 .

\section{Numerical Evaluation}

To show the performance of the prediction scheme (9) and prediction-based control strategy (32), an open-loop unstable system of the form (1)-(4), (6)-(16), (19), (22), (24)-(29), and (31) will be considered, which is determined by

$$
\begin{aligned}
A & =\left[\begin{array}{ll}
0 & 1 \\
0 & 0
\end{array}\right], \\
B & =\left[\begin{array}{l}
0 \\
1
\end{array}\right], \\
C & =\left[\begin{array}{ll}
1 & 0
\end{array}\right], \\
\Phi_{1}(x) & =0.1 \sin \left(x_{2}\right)
\end{aligned}
$$

with a time delay $\tau=0.1$.

To design predictor (9) it was considered that $L=$ $\left[\begin{array}{ll}4.75 & 5.625\end{array}\right]^{\top}$ that set the poles of matrix $A-L C$ in $\{-2.25,-2.5\}$. It was considered that $W_{0}=\operatorname{diag}\{0.79\}, W_{1}=$ $\operatorname{diag}\{2.1\}$ from where $\lambda_{\text {min }}\left(W_{0}\right)=0.79, \lambda_{\text {min }}\left(W_{1}\right)=2.1$.

From the parameters of the system the following bounds are computed: $\gamma_{L}=0.1$ and $\left\|A_{1}\right\|=\|-L C\|=7.3622$. The bound $v=\max _{\theta \in[0, \tau]}\|U(\theta)\|$ is obtained from the time evolution of matrix $U(\theta)$, which can be numerically computed from the procedure proposed in [40]. The evolution of $U(\theta)$ is depicted in Figure 1; thus one has $v=1.2597$.

Finally, it is obtained that $(i) \tau<0.1045$, (ii) $\lambda_{\min }\left(W_{1}\right)>$ 0.9274 , which satisfy the conditions of the Theorem 3 . For all

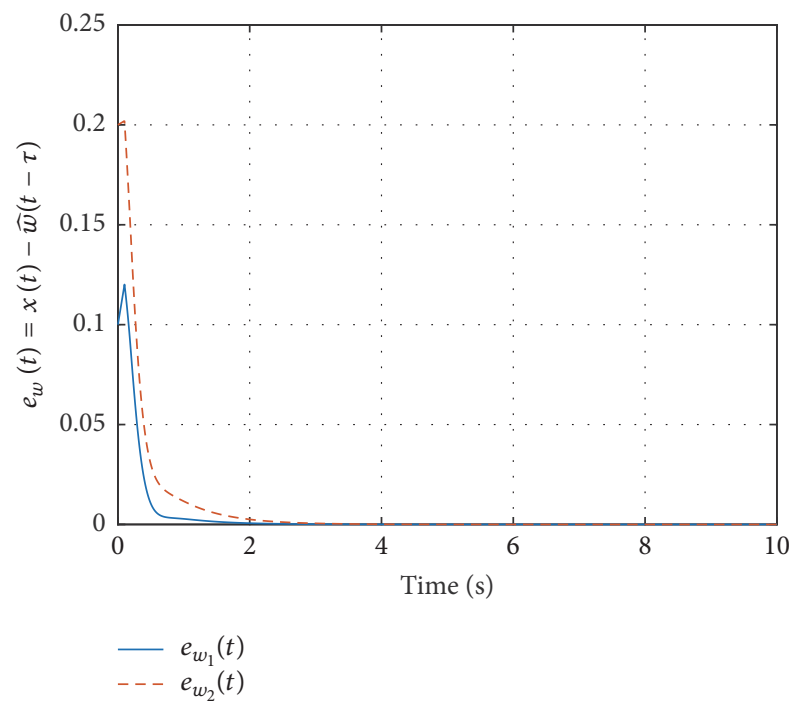

Figure 2: Prediction-observation errors $e_{w}(t)$.

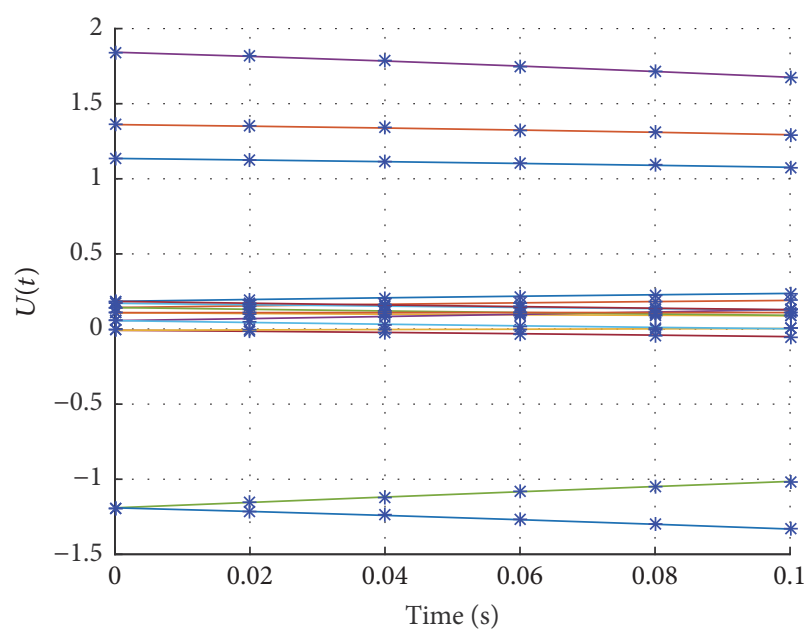

FIGURE 3: Evolution of matrix $U_{r}(t)$.

the experiments, it was considered that $x(0)=\left[\begin{array}{ll}0.1 & 0.2\end{array}\right]^{\top}$. The prediction error evolution $e_{w}(t)=x(t)-\widehat{w}(t-\tau)$ is shown in Figure 2 where its convergence to the origin is clearly stated.

To stabilize system (63) at the origin, the predictor described before was considered and $k=\left[\begin{array}{ll}-3.5 & -3.75\end{array}\right]$ that set the poles of $A+B k$ in $\{-1.75,-2\}$. Notice that in this case $W_{0 r}=\operatorname{diag}\{0.79\}, W_{1 r}=\operatorname{diag}\{2.1\}$ from where $\lambda_{\min }\left(W_{0 r}\right)=$ $0.79, \lambda_{\text {min }}\left(W_{1 r}\right)=2.1, \gamma_{L r}=0.1414$, and $\left\|A_{1 r}\right\|=7.3622$. The computation of $U_{r}(\theta)$ is depicted in Figure 3 from where $v=2.8232$. Notice that the conditions of Theorem 7 are satisfied. The evolution of the state $x(t)$ is depicted in Figure 4 where appropriate convergence to the origin is obtained.

6.1. Trajectory Tracking Problem Based on Predicted State. To assess the flexibility of the proposed control scheme, notice that feedback of the form

$$
\begin{aligned}
u(t)= & -k_{1}\left(\widehat{w}_{1}-w_{1 d}\right)-k_{2}\left(\widehat{w}_{2}-w_{2 d}\right)+\dot{w}_{2 d} \\
& -0.1 \sin \left(\widehat{w}_{2}\right)
\end{aligned}
$$




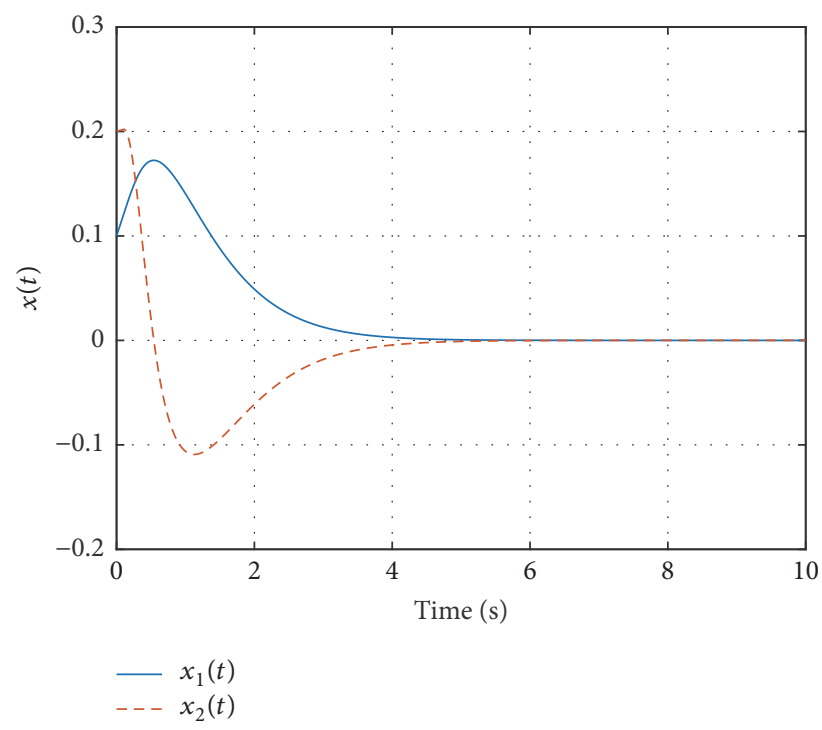

Figure 4: Time evolution of state $x(t)$.

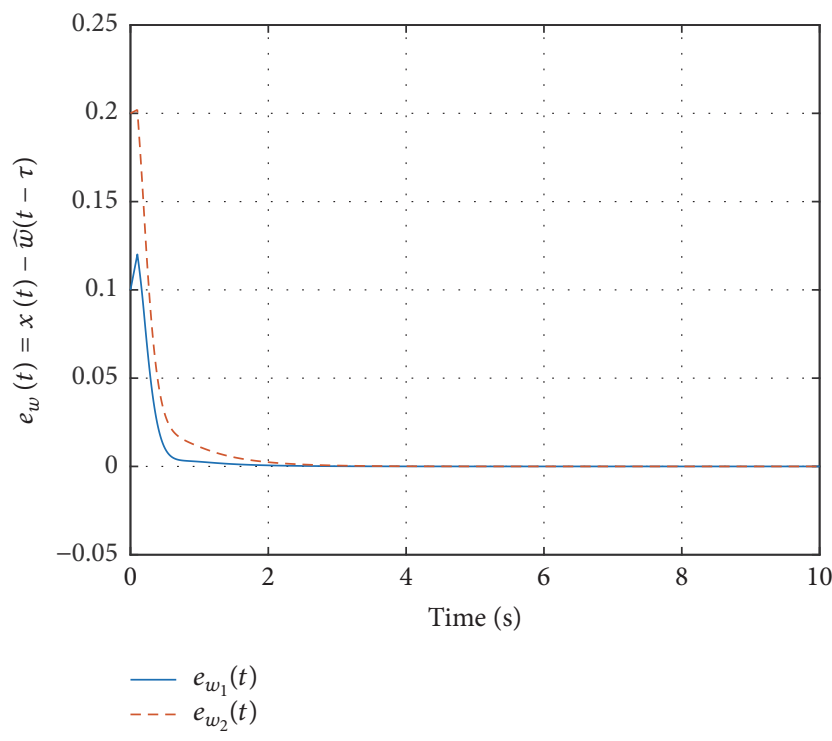

Figure 5: Prediction-observation errors $e_{w}(t)$.

for $k_{i}$ defined as in the stabilization problem solves the trajectory tracking problem associated with (63), for a differentiable desired reference:

$$
\begin{aligned}
w_{d}(t) & =\left[\begin{array}{ll}
w_{1 d}(t) & w_{2 d}(t)
\end{array}\right]^{\top} \\
& =\left[\begin{array}{ll}
x_{1 d}(t+\tau) & x_{2 d}(t+\tau)
\end{array}\right]^{\top}
\end{aligned}
$$

given as $x_{1 d}(t)=A \sin (\omega t), x_{2 d}(t)=A \omega \cos (\omega t)$ for $A=1$, $\omega=2 \pi f, f=0.125$.

The prediction errors are shown in Figure 5 while the tracking errors are depicted in Figure 6; both errors converge to the origin as expected. The corresponding predictorbased feedback is shown in Figure 7. Finally, the estimated perturbation signal is shown in Figure 8.

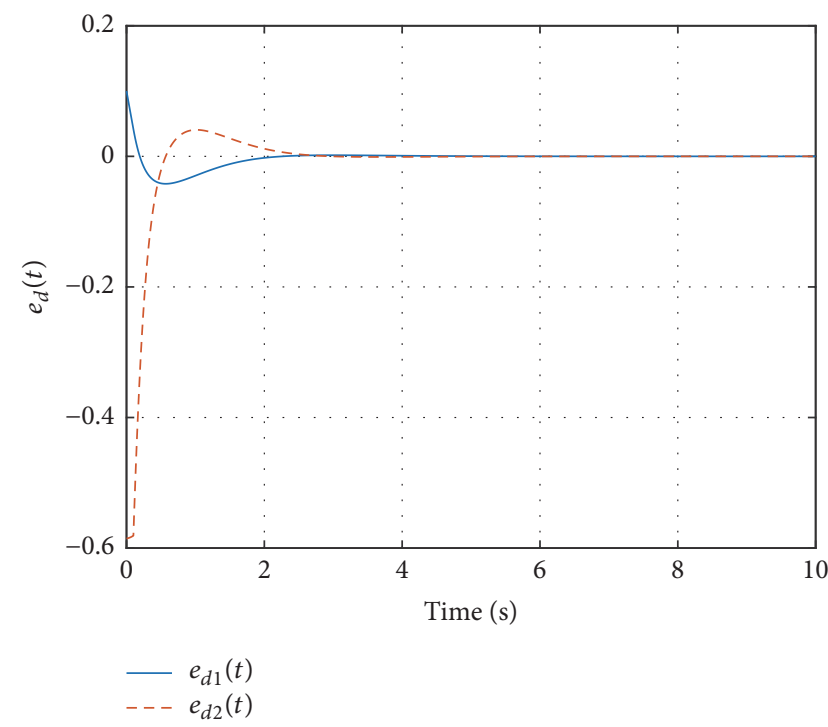

FIgURE 6: Tracking error $e_{d}(t)$.

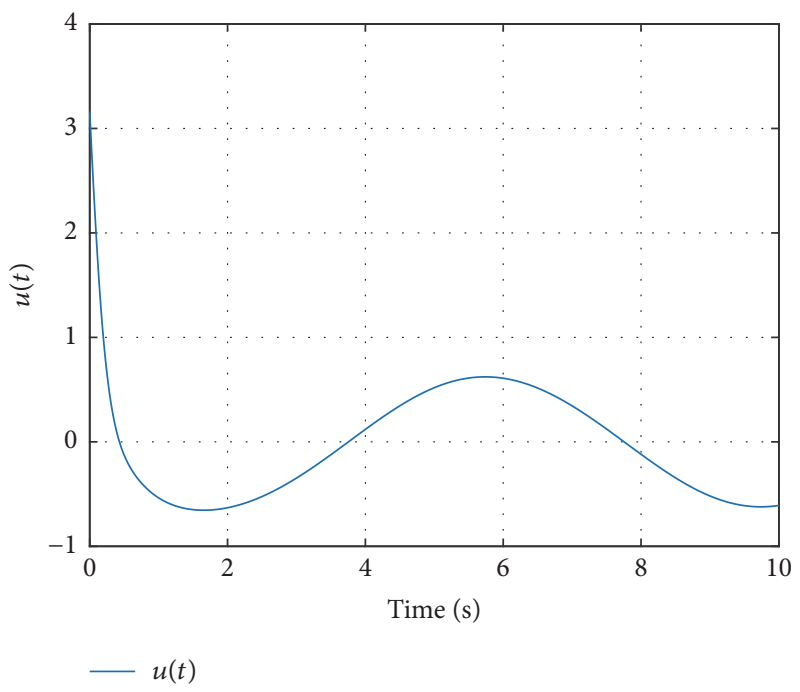

FIGURE 7: Control signal $u(t)$.

\section{Conclusions}

This work addresses and provides solutions to the state prediction and control problems of a class of nonlinear systems with known constant time delay at the input signal. An appropriate time shift of the state coordinates lets the predictor be designed as an observer. The predictor convergence depends on the time delay magnitude and the Lipschitz constant associated with the class of considered systems. The future estimated states solve the regulation and trajectory tracking problems. Numerical simulations evaluate the prediction strategy as well as the regulation and trajectory tracking controller based on predicted states.

Additionally, it is shown that the presented predictorcontrol strategy also applies to the class of full linearizable nonlinear systems. As a continuation of this work, it is 


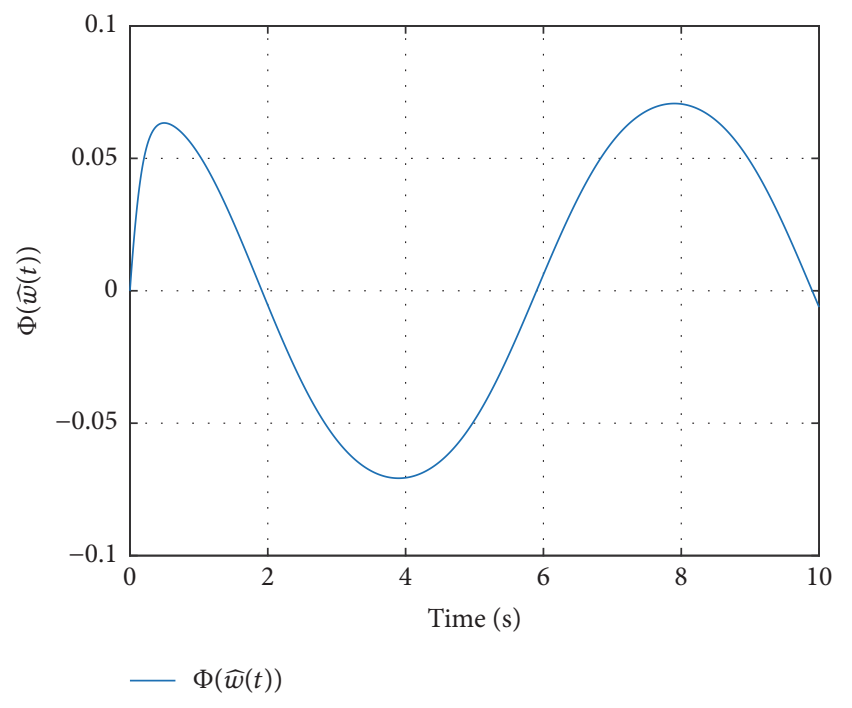

Figure 8: Perturbation signal $\Phi(\widehat{w}(t))$.

important to consider the increase in the time delay that the closed loop system can handle. Also, going a little bit further, the time varying delay case and the real-time implementation of the proposed algorithm should be boarded.

\section{Conflicts of Interest}

The authors declare that there are no conflicts of interest regarding the publication of this paper.

\section{Acknowledgments}

The work of the first and second author was partially supported by Conacyt, Mexico, under Grant CB-254329.

\section{References}

[1] E. Fridman, Introduction to Time-Delay Systems: Analysis and Control, Birkhäuser, Basel, Switzerland, 2014.

[2] V. Kolmanovskii and A. Myshkis, Applied theory of functional differential equations, Kluwer Academic Publishers, Dordrecht, The Netherlands, 1992.

[3] J. K. Hale, Theory of Functional Differential Equations, SpringerVerlag, NY, USA, 1997.

[4] S. I. Niculescu, Delay Effects on Stability: A Robust Control Approach, vol. 269, Springer Science \& Business Media, Berlin, Germany.

[5] O. J. M. Smith, "Closer control of loops with deadtime," Chemical Engineering Progress, vol. 53, no. 5, pp. 217-219, 1957.

[6] Z. J. Palmor, "Time-delay compensation Smith predictor and its modifications," The Control Handbook, vol. 1, pp. 224-229, 1996.

[7] A. Germani, C. Manes, and P. Pepe, "A new approach to state observation of nonlinear systems with delayed output," Institute of Electrical and Electronics Engineers. Transactions on Automatic Control, vol. 47, no. 1, pp. 96-101, 2002.

[8] M. Hou, P. Ztek, and R. J. Patton, "An observer design for linear time-delay systems," Automatic Control, vol. 47, no. 1, pp. 121$125,2002$.
[9] F. E. Thau, "Observing the state of nonlinear dynamic systems," International Journal of Control, vol. 17, no. 3, pp. 471-479, 1973.

[10] R. Rajamani, "Observers for Lipschitz nonlinear systems," Institute of Electrical and Electronics Engineers. Transactions on Automatic Control, vol. 43, no. 3, pp. 397-401, 1998.

[11] F. Mazenc and P.-A. Bliman, "Backstepping design for timedelay nonlinear systems," Institute of Electrical and Electronics Engineers. Transactions on Automatic Control, vol. 51, no. 1, pp. 149-154, 2006.

[12] I. H. Kim and Y. I. Son, "Robust control for input time-delay systems: a disturbance observer approach," in Proceedings of The SICE Annual Conference 2010, pp. 179-183, IEEE, Taipei, Taiwan.

[13] Z.-Y. Li, B. Zhou, and J. Lam, "Lyapunov-Krasovskii functionals for predictor feedback control of linear systems with multiple input delays," Applied Mathematics and Computation, vol. 244, pp. 303-311, 2014.

[14] Y. H. Roh and J. H. Oh, "Sliding mode control with delay compensation for uncertain input-delay systems," American Control Conference, vol. 1, pp. 309-313, 1999.

[15] B. Lee and J. G. Lee, "Robust control of uncertain systems with input delay and input sector nonlinearity," in Proceedings of the 39th IEEE Conference on Decision and Control, vol. 5, pp. 44304435, IEEE, Sydney, Australia.

[16] C. C. Chiang and T. C. Tung, "Robust tracking control of uncertain nonlinear systems with an input time delay," in Proceedings of the 2003 International Joint Conference on Neural Networks, vol. 3, pp. 2394-2399, Portland, OR, USA, July 2003.

[17] J. Lei and H. K. Khalil, "Feedback linearization for nonlinear systems with time-varying input and output delays by using high-gain predictors," Institute of Electrical and Electronics Engineers. Transactions on Automatic Control, vol. 61, no. 8, pp. 2262-2268, 2016.

[18] M. Krstic, "Lyapunov stability of linear predictor feedback for time-varying input delay," Institute of Electrical and Electronics Engineers. Transactions on Automatic Control, vol. 55, no. 2, pp. 554-559, 2010.

[19] A. Z. Manitius and A. W. Olbrot, "Finite spectrum assignment problem for systems with delays," Institute of Electrical and Electronics Engineers. Transactions on Automatic Control, vol. 24, no. 4, pp. 541-553, 1979.

[20] M. Krstic, Delay Compensation for Nonlinear, Adaptive, and PDE Systems, Springer, Berlin, Germany, 2009.

[21] W. Michiels, K. Engelborghs, P. Vansevenant, and D. Roose, "Continuous pole placement for delay equations," Automatica. A Journal of IFAC, the International Federation of Automatic Control, vol. 38, no. 5, pp. 747-761, 2002.

[22] W. Michiels, S. Mondie, and D. Roose, Robust stabilization of time-delay systems with distributed delay control laws: necessary and sufficient conditions for a safe implementation, Department of Computer Science, K.U.Leuven, Leuven, Belgium, 2003.

[23] L. Mirkin, "On the approximation of distributeddelay control laws," System Control Letters, vol. 51, pp. 331-342, 2004.

[24] S. Mondié and W. Michiels, "Finite spectrum assignment of unstable time-delay systems with a safe implementation," IEEE Transactionson Automatic Control, vol. 48, no. 12, pp. 2207-2212, 2003.

[25] B. Zhou, Z. Lin, and G.-R. Duan, "Truncated predictor feedback for linear systems with long time-varying input delays," Automatica. A Journal of IFAC, the International Federation of Automatic Control, vol. 48, no. 10, pp. 2387-2399, 2012. 
[26] Z. Zuo, Z. Lin, and Z. Ding, “Truncated prediction output feedback control of a class of lipschitz nonlinear systems with input delay," IEEE Transactions on Circuits and Systems II: Express Briefs, vol. 63, no. 8, pp. 788-792, 2016.

[27] W. Michiels and D. Roose, "Time-delay compensation in unstable plants using delayed state feedback," in Proceedings of the 40th Conference on Decision and Control, vol. 2, pp. 14331437, IEEE, Orlando, FL, USA, Dec. 2001.

[28] K. Shi, X. Liu, Y. Tang, H. Zhu, and S. Zhong, "Some novel approaches on state estimation of delayed neural networks," Information Sciences, vol. 372, pp. 313-331, 2016.

[29] K. Shi, X. Liu, H. Zhu, S. Zhong, Y. Zeng, and C. Yin, "Novel delay-dependent master-slave synchronization criteria of chaotic Lur'e systems with time-varying-delay feedback control," Applied Mathematics and Computation, vol. 282, pp. 137-154, 2016.

[30] V. L. Kharitonov, Time-Delay Systems: Lyapunov Functionals and Matrices, Birkhäuser, Boston, Mass, USA, 2013.

[31] S. Raghavan and J. K. Hedrick, "Observer design for a class of nonlinear systems," International Journal of Control, vol. 59, no. 2, pp. 515-528, 1994.

[32] S. R. Kou, D. L. Elliott, and T. J. Tarn, "Exponential observers for nonlinear dynamic systems," Information and Control, vol. 29, no. 3, pp. 204-216, 1975.

[33] M. Velasco-Villa, J. Heras-Godnez, J. A. Vázquez-Santacruz, and V. Fragoso-Rubio, "Delayed consensus problem for single and double integrator systems," Mathematical Problems in Engineering, vol. 2015, Article ID 461098, 15 pages, 2015.

[34] M. Velasco-Villa, R. Castro-Linares, F. Rosales-Hernández, B. Del Muro-Cuellar, and M. A. Hernández-Pérez, "Discrete-time synchronization strategy for input time-delay mobile robots," Journal of the Franklin Institute, vol. 350, no. 10, pp. 2911-2935, 2013.

[35] J. Ordaz, S. Salazar, S. Mondié, H. Romero, and R. Lozano, "Predictor-based position control of a quad-rotor with delays in GPS and vision measurements," Journal of Intelligent and Robotic Systems: Theory and Applications, vol. 70, no. 1-4, pp. 13-26, 2013.

[36] K. Gu, J. Chen, and V. L. Kharitonov, Stability of time-delay systems, Springer Science Business Media, Berlin, Germany.

[37] R. E. Bellman and K. L. Cooke, Differential-Difference Equations, Rand Corporation, Santa Monica, Calif, USA, 1963.

[38] R. V. Patel and M. Toda, "Quantitative measures of robustness in multivariable systems," Joint Automatic Control Conference, 1980.

[39] A. Isidori, Nonlinear Control Systems, Springer-Verlag, London, UK, 3rd edition, 1995.

[40] H. Garcia-Lozano and V. L. Kharitonov, "Numerical computation of time delay Lyapunov matrices," IFAC Proceedings Volumes, vol. 39, no. 10, pp. 60-65, 2006. 


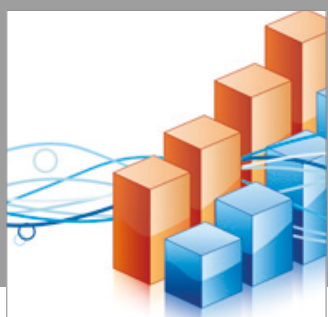

Advances in

Operations Research

vatersals

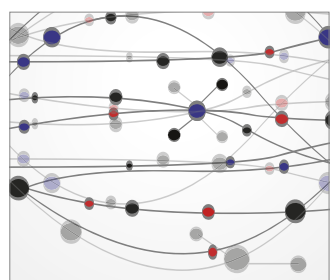

\section{The Scientific} World Journal
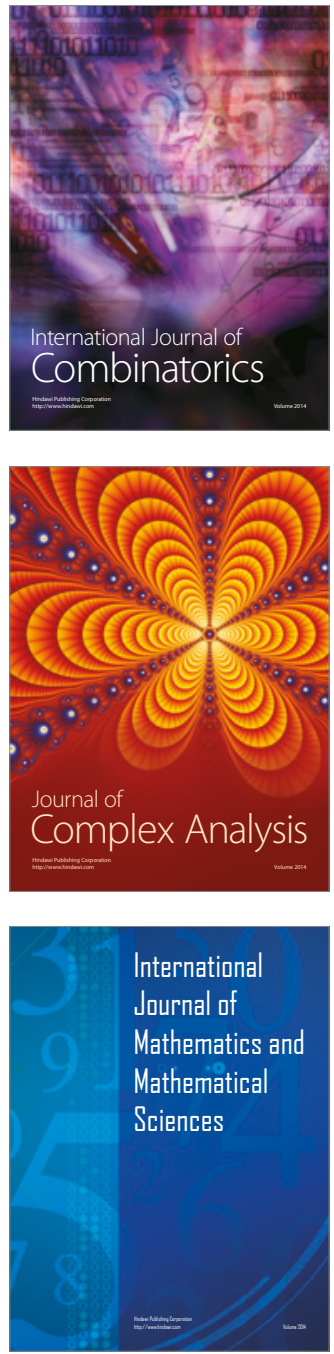
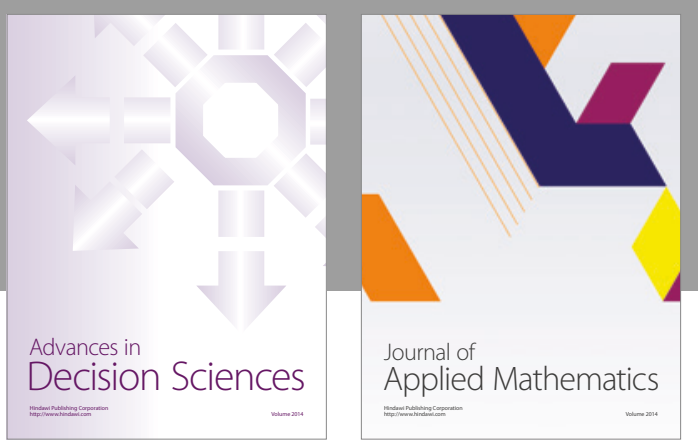

Algebra

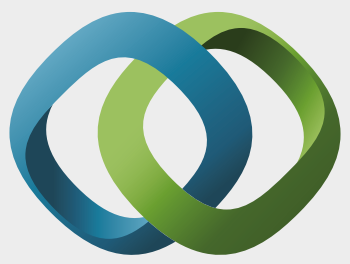

\section{Hindawi}

Submit your manuscripts at

https://www.hindawi.com
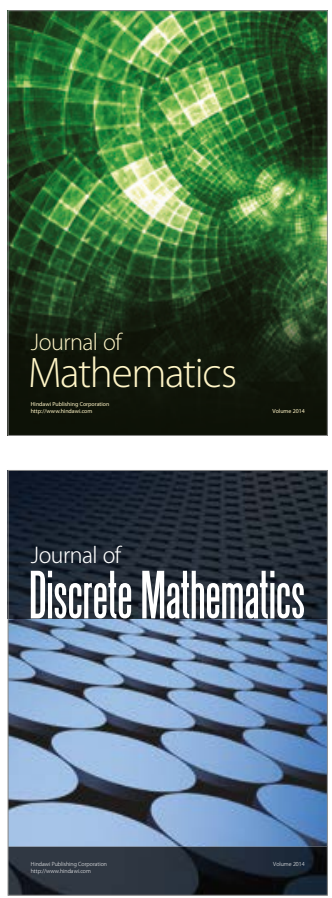

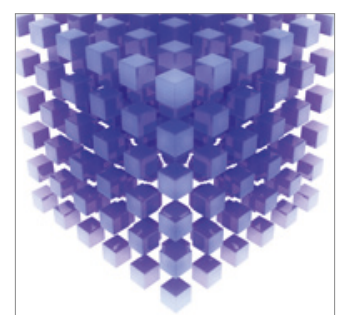

Mathematical Problems in Engineering
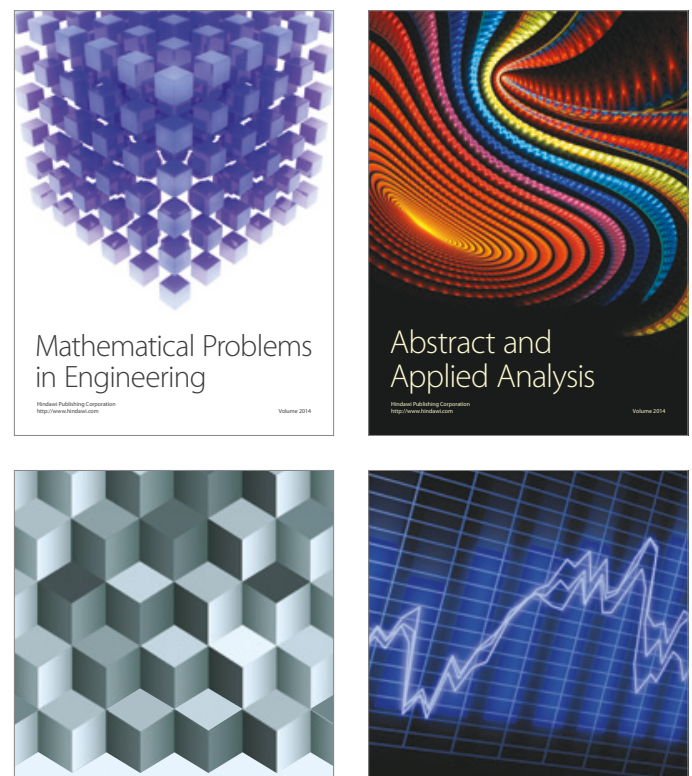

Journal of

Function Spaces

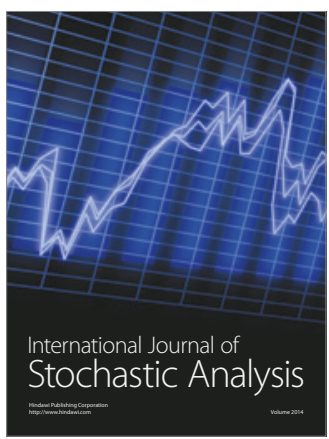

Probability and Statistics
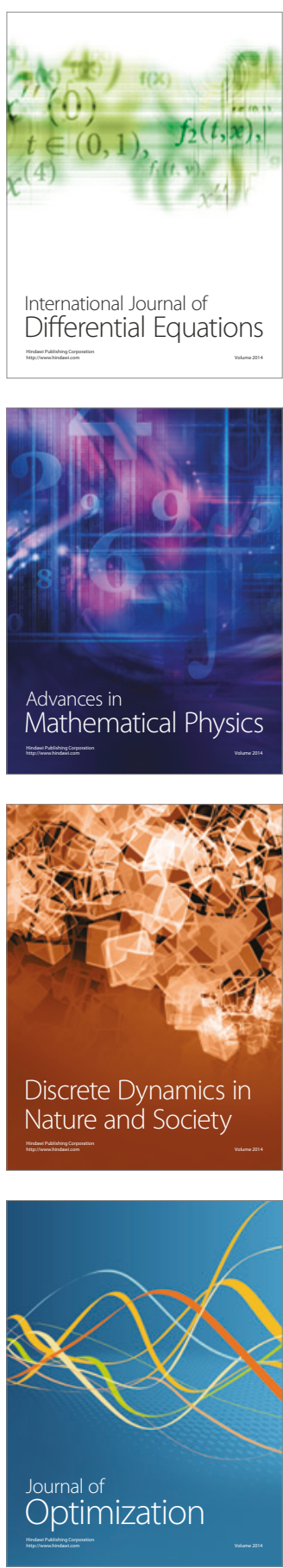